\title{
Gym Monitoring Framework for Fitness Management System
}

\author{
Mr Akshay Sambare ${ }^{1}$, Dipali Bondre ${ }^{1}$, Sachin Thorat ${ }^{1}$, Miss Archana Vishe ${ }^{1}$, Prof. Ankit Sanghavi ${ }^{2}$ \\ UG Scholar, Dept of Computer Science, Alamuri Ratnamala Institute of Engineering and Technology, Mumbai, India ${ }^{1}$ \\ Asst Prof, Dept of Computer Science, Alamuri Ratnamala Institute of Engineering and Technology, Mumbai, India ${ }^{2}$
}

\begin{abstract}
Usually, the client uses MS Excel or paper, and maintains their records, however it is not possible for them to share the data from multiple system in multi user environment, there is lot of duplicate work, and chance of mishap. Every Excel file need to be updated once records are been changed The Fitness Freak System rejects most of the disadvantages of the existing software. Increasing efficiency and effectiveness, automation, accuracy, user-friendly interface, information availability, communication capacity, maintenance, cost deduction makes our system smarter than the existing system. We intermingle some new and blatant features along with all the necessary features. Some of them are user login, platform independent, etc...
\end{abstract}

Keywords: Gym Monitoring Framework, Fitness Management System, login, platform independent, MS Excel.

\section{INTRODUCTION}

Fitness Freak enables the effortless scheduling of training resources, massage facility, appointment with Nutritionist, and generation of fitness reports. The system helps manage meetings with various resources, giving details of the involved members and employees in charge.The members of the gym can be classified on the basis of their membership programmers and they can be assisted in planning out their diet if needed.Based on the calorie need of individual diet is been categorized and alloted accordingly. The system enables generation of exercise cards, fitness reports for each member to track individual member progress.

This system not only evaluates all the information of each and every member but it also gives us to future updations and also this system showing a messaging system where user can easily understand about it. Fitness Freak allows the gym to gain significant time benefit, enhance productivity and cost-savings, allowing the owner to better monetize your gym's resources and staff, thus helping to improve the overall profitability. The system of Gym Management also included complaints and feedback from our employee base and user base to greatly develop our system features. Fitness Freak provides a wide range of reporting on members and programmers.

Everyone knows that health is a wealth and without a health We do not need a anything which give us happiness so that's why healthy personality is a most important thing we need to keep in our mind.in a normal and secure life view our energy to do anything is got through healthy and fitness of our body. For a healthy and tension free life a Physical fitness is very necessary and important. Physical fitness includes exercise and sleep, diet. there are three basic things have own importance in each individual and personal life and everyone should be sensible with regard to these for a healthy, fit as well as happy life .
Fitness Freak is a gym and health club membership management system. It is a system designed to manage membership data files, employee records and their related functions. Fitness Freak enables the effortless scheduling of appointment with Nutritionist, massage facility, training resources and creation reports of fitness. The system helps manage appointments with various resources, giving details of the involved members and employees in charge. The members of the gym can be classified on the basis of their membership programs and they can be assisted in planning out their diet if needed. Diets are classified based on the calorie needs of an individual. The system enables generation of exercise cards, fitness reports for each member to track individual member progress.

fitness freak system not only encapsulate all the important information of each and every member but it also provides for future updations and also provide a user login as well as manager login for security purpose. Fitness Freak allows the enhance productivity and cost-savings, gym to gain significant time benefit, allowing the owner to better monitoring your gym's resources and staff provide owner login for handling system, thus helping to develop and improve the overall profitability and ability of system. Our fitness freak System also included messaging, mail to customer feed-back, complaints from our employee base and user base to develop our system features.

Fitness Freak provides a wide range of reporting on members and programs. Fitness Freak helps the gym to retain members, save time, give better service and track its performance, keep information.

\section{FEASIBILITY STUDY REPORT}

The feasibility of a project can be considered in terms of technical factors, economic factors or both. A feasibility 
study includes documents with a report showing all the features of the project. Our software Fitness Freak includes the following feasibility analysis:

\section{Technical Feasibility}

Technical feasibility refers to the adroitness of the process to take benefit of the current state of the technology in pursuing further improvements. Fitness Freak is developed in java. The object oriented feature which java supports is our requirement. Java was preferred over other languages because it is platform independence as well as object oriented feature.

\section{Operational Feasibility}

There are two aspects to check the operational feasibility of the system. One is the technical performance and the other one is acceptance. Technical performance deals with the fact that whether the system provides correct and timely data required for the end user.

3. Economic Feasibility

The economic feasibility of the system deals with its financial aspects and determines whether the project is economically feasible or not.

\section{EXISTING SYSTEM}

An Existing system refers to the system that is being followed till now. The gym is working manually. The current system is taking more time and also it is very costly, because it includes a lot of paperwork. To manually handle the system was very difficult task. But now-a-days computerization made easy to work.

The following are the reasons why the current system should be computerized:

-To increase efficiency with reduced cost.

- To reduce the burden of paper work.

-To save time management for recording details of each and every member and employee.

-To generate required reports easily.

\section{PROPOSED SYSTEM}

The software will help the user in following ways:

Fitness Freak saves time by providing easy search operations.

I.Provides an easy to use interface through which user can directly store information in the database.

ii.Provides access to different programs and additional facilities for the members.

iii.Exercise card generation helps the members to follow a particular set of exercise depending on the type of program selected.

iv.Expiry lists are generated to identify members whose memberships are expiring.

V.Unlimited user definable reports to analyze sales, profits, number of customers using a particular program, expense report, etc.

vi.Fitness freak is easy to customize and flexible for any changes to be made in the future.
vii.It measures the member progress by generating fitness reports at regular intervals which helps in achieving the member goals

viii.Helps retains customers by giving birthday reminders, payment reminders, and appointment reminders.

ix.Fitness freak also has facilities to easily update information if the information is incorrect.

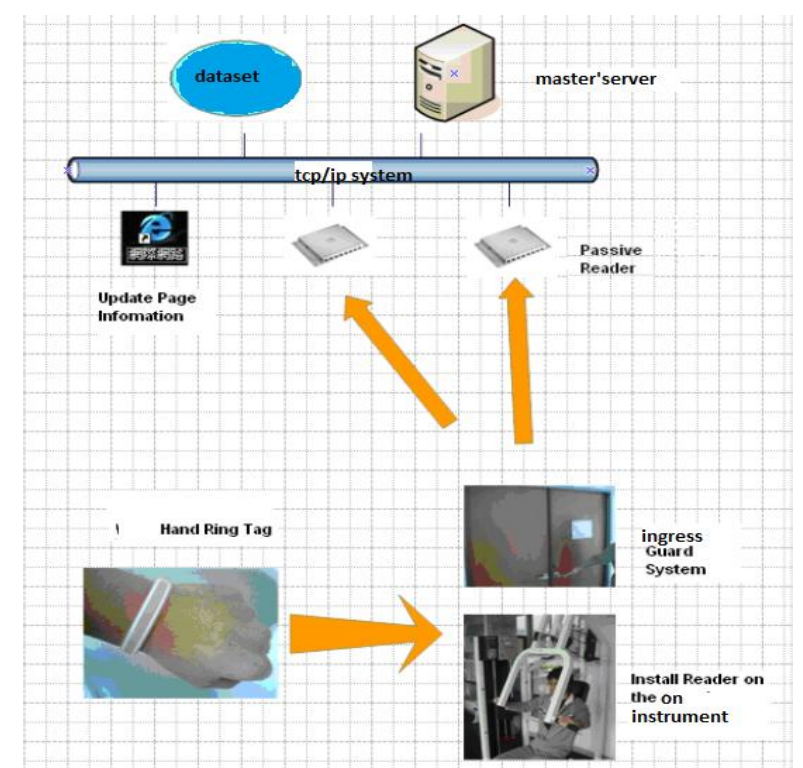

FIG.1.1 SYSTEM ARCHITECTURE

\section{CONCUSION}

As per the overall implementation of the system and various modules discussed in the project, we can now conclude efficient and highly user-friendly compressor and decompression Software that is easy to implement can be thus devised. Not only is this project highly flexible, but also makes very interesting viewing it is capable of handling a various number of Files for splitting as well as merging a large file. Passing the any kind of file and splitter in to live videos as well as audio does the communication.

\section{REFERENCES}

[1] Real time sentiment analysis of twitter data using Hadoop Sunil B. Mane, Yashwant Sawant, Saif Kazi, Vaibhav Shinde

[2] IEEE paper "Research on Public Opinion Based on Big Data" 1.Songtao Shang, 2.Minyong Shi, 3.Wenqian Shang, 4.Zhiguo Hong

[3] https://hadoop.apache.org/

[4] http://www.tutorialspoint.com/hadoop/

[5] http://www.javatpoint.com/java-tutorial

[6] https://en.wikipedia.org/wiki/Apache_Hadoop 